\title{
TRACEABILITY AND MANAGEMENT OF MEDICINES AND MEDICAL DEVICES AT HOME: THE CRUCIAL ROLE OF THE DIGITAL SUPPORT
}

\author{
Paolo Locatelli, Federica Cirilli, Eliana Panno and Antonio Jacopo Scardigno \\ Fondazione Politecnico di Milano, Italy
}

\begin{abstract}
The continuous growth of the healthcare sector is followed by a corresponding demand to reduce costs and to improve the quality of its management processes. One way to meet these requirements is to leverage on a better management and traceability of medicines and medical devices through ICT, not only at the hospital but also at the patient's home. The project "LIFEMED - Integrated Logistic of Medicines and Medical Devices", aims, indeed, at the realization of an innovative digital model for medicines and medical devices logistic management. In particular, for the management and the traceability of medicines and medical devices at home, the solution will be characterized by a telemedicine platform together with a traceability platform based on RFId and barcode labels and readers. The telemedicine and traceability platforms will allow for a better monitoring of respectively the patient's correct medicine administration and the medicines and medical devices logistic, as demonstrated by the example of the peritoneal dialysis, a domiciliary treatment addressed in the project. Despite all the advantages, attention must be paid during the platform design in considering crucial factors like digital illiteracy of older people and the privacy, legal and ethical issues.
\end{abstract}

\section{KEYWORDS}

Medicines, Medical Devices, Digital Support, Traceability, Peritoneal Dialysis

\section{INTRODUCTION: SCENARIO AND CHALLENGES}

The healthcare sector is currently going through a significant growth, remaining one of the most popular investment theme in developed markets and Countries, especially those with ever increasing ageing population [Lulin et al, 2016]. Nonetheless this growth is followed by a corresponding demand to reduce costs and a continuous pressure to improve the quality of management processes [Agarwal et al, 2010]. One way to meet these requirements is to leverage on a better management and traceability of medicines and medical devices, not only at the hospital, but also at the patient's home. As a matter of fact, having a trustworthy and monitored provision of health services at a distance can result in the costs reduction thanks to a lower patient hospitalization [Paul, 2013]. Moreover, an efficient traceability at a distance can lead to a better management of unused medicines, avoiding uneconomic waste, and to a better prevention of errors and more controlled deployment of medical devices [Lovis, 2008] [Matta et al, 2013].

In this regard, digital technologies proved to play a crucial role in the processes management and organizational evolution of medicines and medical devices [Geneva: World Health Organization; 2019].

\section{LIFEMED DIGITAL SOLUTION}

The project "LIFEMED - Integrated Logistic of Medicines and Medical Devices", funded by the Italian Ministry of Education, Universities and Research (MIUR - Ministero dell'Istruzione, dell'Università e della Ricerca), aims at the realization of an innovative organizational-technological model for medicines and medical devices logistic management at the hospital and at home. In particular, the overall solution (Figure 1) is conceived as a digital and modular platform that enables the clinical governance and the monitoring of all the processes related to medicines and medical devices, leveraging on virtual warehouse management, risk 
management, decision support system and medicines prescription and recovery/administration management in and out the hospital. The solution integrates also external existing systems in order to avoid a double-input of information by the hospital staff.

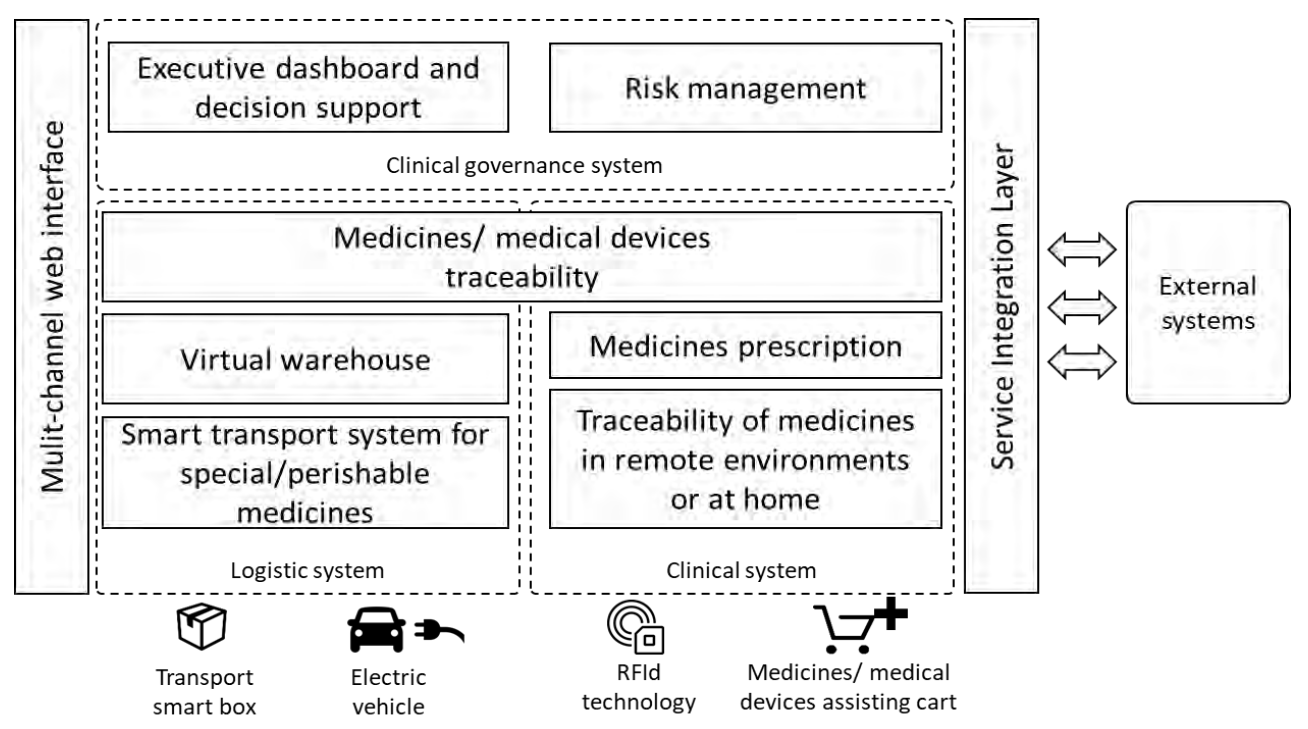

Figure 1. LIFEMED solution

Regarding specifically the management of medicines and medical devices at home, the solution will consist of a client-to-provider telemedicine platform that will enable the patient to correctly perform the medicine administration. The patient, indeed, will be provided with a personal account (accessible through a smartphone, a tablet or a web-based user interface) that will allow him to: receive automatic reminders (e.g. time and dosage) about taking the medicine and notify that the administration has been carried out correctly or enter supporting notes when deciding not to carry it out. Doctors or other members of the medical team will be able to access the monitoring telemedicine platform in order to consult the history of the treatments carried out and view any warnings of non-administration.

Furthermore, the solution will also include a traceability platform so as to monitor all medicines and medical devices movements during transportation to the patient's home. The traceability system will be based on RFId labels technology to track medical devices and special medicines and on barcode technology to track generic medicines, coupled with portable devices provided with RFId and barcode readers.

The LIFEMED platform efficiency in management and traceability of medicines and medical devices at home can be highlighted through the application in a specific treatment: the peritoneal dialysis (PD).

\section{CASE STUDY: PERITONEAL DIALYSIS (PD)}

PD is a treatment option that enables patients suffering from end-stage renal disease to perform dialysis in their own home thus allowing them to have a higher quality of life [Russo et al, 2006]. Despite its diffusion, PD is still affected by several issues: inadequate and not repeated patients' trainings, trainings require more time than most nephrologist can provide, trainings effectiveness should be periodically tested, high noncompliance rate among patients and waste of domiciliary medicines that are not correctly traced back [Novak et al, 2008] [Kazancioglu et al, 2008]. In many cases, all these problems drive patients not to feel confident in adopting PD or the treatment's inadequate application can cause severe patients' complications leading to their hospitalization as a consequence of the switching from PD to haemodialysis [Fried et al, 1999].

The analysed as-is management process for PD in a clinical centre involved in the LIFEMED project can widely reflect some of the abovementioned issues. In this case, all the medicines are considered as used by the hospital pharmacy as soon as they are delivered to the patient's home. Moreover, patient's education about manual PD is provided mainly at the hospital site. Finally, when a manual PD is provided (without an 
automated dialysis medical device), there are no reliable information regarding the efficiency and periodicity of the patient's treatment: the patient can only report his/her information in a paper form during periodical visits at the outpatient clinic of the hospital.

LIFEMED solution will provide concrete evidence to tackle these problems allowing for the monitoring of the patient's correct adherence to the treatment. Moreover, the partners are evaluating the chance to introduce some functionalities on the telemedicine platform in order to permit the patient to enter his/her vital signs periodically recorded (e.g. heartbeat, pressure) and consult digital training materials (e.g. digital brochure, video and audio tutorials). Furthermore, RFId and barcode traceability will allow the hospital pharmacy to avoid the waste of unused medicines, that can be transferred from the patient's home to the hospital as warehouse stocks, and will also allow a more efficient tracing of faulty products.

\section{CONCLUSIONS}

The project is still ongoing and now is focused on analysing the as-is processes and governance in the involved clinical centres. The partners are also currently involved in designing the new process-organizational model and the related support through the integrated digital platform. In particular, they are focusing on the technical design and completion (hardware and software) related to the RFId traceability and to both the telemedicine and traceability platforms. Crucial attention must be paid in considering digital illiteracy of older people, the wide heterogeneity of the processed data, the use of standardized RFId carriers and readers and all the privacy, legal and ethical issues involved in the traceability [Lovis, 2008] [AbuKhousa et al, 2014].

Once the designing is completed, the system applications components will be developed and, finally, the project results will be tested and monitored in the pilot centres in order to have information to define an innovative business model 0 for the medicines and medical devices logistic.

\section{REFERENCES}

AbuKhousa E. et al, 2014. Simulation and modeling efforts to support decision making in healthcare supply chain management. The Scientific World Journal, Vol. 2014, Article ID 354246, 16pp.

Agarwal R. et al, 2010. Research Commentary-The Digital Transformation of Healthcare: Current Status and the Road Ahead. Information Systems Research, 21(4): 796-809.

Fried L. et al, 1999. Hospitalization in peritoneal dialysis patients. American Journal of Kidney Diseases, 33(5): 927-933.

Geneva: World Health Organization; 2019. WHO guideline: recommendations on digital interventions for health system strengthening.

Kazancioglu R. et al, 2008. Importance of Home Visits in Peritoneal Dialysis. Dialysis \& Transplantation, 1-3.

Lovis C., 2008. Traceability in Healthcare: Crossing Boundaries. IMIA Yearbook of Medical Informatics, 47 Suppl $1: 105-113$

Lulin Z. et al, 2016. The effect of herd formation among healthcare investors on health sector growth in China. International Journal for Equity in Health, 15:116.

Matta A. et al, 2013. A Decision-Making Approach Supporting Hospital Drug Logistics. Proceedings of the International Conference on Health Care Systems Engineering. Springer Science \& Business Media, (19): - 241-252.

Novak M. et al, 2008. Why Is Peritoneal Dialysis Underutilized in the United States? Dialysis \& Transplantation, $1-2$.

Paul D. P., 2013. An Innovation in Healthcare Delivery: Hospital at Home. Journal of Management Policy and Practice, 14(6): 73-91.

Pistorio A. et al, 2017. A Business Model for Digital Healthcare Environments: An Organic Approach and a Use Case for Handling Cognitive Impairment. Proceedings of the 10th International Joint Conference on Biomedical Engineering Systems and Technologies HEALTHINF, BIOSTEC, pp. 340-347.

Russo R. et al, 2006. Patient re-training in peritoneal dialysis: Why and when it is needed. Kidney International, 70: S127-S132. 